\title{
Efeitos do Consumo Agudo de Cafeína sobre Parâmetros Metabólicos e de Desempenho em Indivíduos do Sexo Masculino
}

\section{Effects of Caffeine Acute Consumption on the Metabolic And Performance Parameters in Male Individuals}

Daniela Brunetto'

Jerri Luiz Ribeiro²

Ana Paula Trussardi Fayh ${ }^{3}$

1. Acadêmica do Curso de Nutrição do Centro Universitário Metodista IPA - Porto Alegre, RS.

2. Professor do Curso de Fisioterapia do Centro Universitário Metodista IPA - Porto Alegre, RS.

3. Professora dos Cursos de Nutrição e Educação Física do Centro Universitário Metodista IPA - Porto Alegre, RS.

\section{Endereço para correspondência:} Rua Cel. Joaquim Pedro Salgado, 80 - Rio Branco - 90420-060 Porto Alegre, RS, Brasil. E-mail: apfayh@yahoo.com.br

\begin{abstract}
RESUMO
O objetivo deste estudo foi avaliar o efeito do consumo agudo de cafeína sobre a oxidação de lipídeos e desempenho durante o exercício aeróbico. Foram avaliados 15 indivíduos do sexo masculino, com idade média de 22,3 $\pm 2,7$ anos, que realizaram teste de cargas progressivas em esteira rolante para determinação do consumo máximo de oxigênio $\left(\mathrm{VO}_{2 \text { máx }}\right)$ e limiares ventilatórios (LV). Cada voluntário realizou três testes submáximos na intensidade de 10\% abaixo do segundo LV, sendo orientados a permanecer em exercício até a exaustão. Trinta minutos antes de cada teste submáximo, foram ingeridos $250 \mathrm{ml}$ de uma das bebidas compostas por: café com adoçante (CAD), café com açúcar (CA) e café descafeinado com adoçante (CD). Durante o exercício, os indivíduos foram monitorados pelo ergoespirômetro e frequencímetro. A oxidação de lipídeos foi predita pelo quociente respiratório (QR) durante o teste, e o desempenho foi verificado pelo tempo de exercício. Para comparar os resultados de QR e tempo de exercício entre os grupos, foi utilizado Anova fatorial, e considerou-se significância estatística um valor de $p<0,05$. A média de $\mathrm{VO}_{2 \text { máx }}$ foi de 50,18 $\pm 9,9 \mathrm{ml} / \mathrm{kg} / \mathrm{min}$. Com a ingestão de CAD, a média do QR foi de 0,98 $\pm 0,18$ e o tempo médio em exercício foi de 24,1 $\pm 17,04$ minutos. Com a ingestão de CA, a média do QR foi de 0,96 \pm 0,2 e o tempo médio em exercício foi de 24,4 $\pm 17,8 \mathrm{~min}$. No teste com ingestão de $C D$, a média do QR foi de 1,01 $\pm 0,24$ e a média do tempo em exercício foi de $20,6 \pm 9,7 \mathrm{~min}$. Não houve diferença significativa entre os testes nos valores do QR e nem tempo de exercício ( $p=0,697$ e $p=0,598$, respectivamente). A cafeína não aumentou a oxidação de lipídeos nem o desempenho de indivíduos jovens do sexo masculino.
\end{abstract}

Palavras-chave: café, exercício aeróbico, recurso ergogênico.

\begin{abstract}
The purpose of this study was to evaluate the effect of acute caffeine consumption on lipid oxidation and performance during aerobic exercise. Fifteen healthy male individuals, $22.3 \pm 2.7$ years old, performed a progressive test on treadmill for determination of maximal oxygen uptake $\left(\mathrm{VO}_{2 \max }\right)$ and ventilatory thresholds. Each volunteer performed three submaximal tests at the intensity of 10\% below the second ventilatory threshold, being guided to remain on exercise until exhaustion. Thirty minutes before each submaximal test, the subjects ingested $250 \mathrm{ml}$ of one of following drinks: coffee with sweetener (CSW), coffee with sugar (CS) or decaffeinated coffee with sweetener (CD). During the exercise, the individuals's heart rate was monitored and respiratory gases analyses were done. The lipid oxidation was predicted by the respiratory quotient (RQ) during the test and performance was verified by exercise duration. In order to compare the RQ results and time of exercise among the three groups, factorial Anova was used, and a value of $p<0.05$ was considered as statistically significant. The individuals had $V_{2 \max }$ of $50.18 \pm 9.9 \mathrm{ml} / \mathrm{kg} / \mathrm{min}$. CAD ingestion caused $\mathrm{RQ}$ average of $0.98 \pm 0.18$, and the average exercise duration was of $24.1 \pm 17.04 \mathrm{~min}$; CA ingestion caused RQ average of $0.96 \pm 0.2$ and the average exercise duration was $24.4 \pm 17.8 \mathrm{~min}$. Finally, CD ingestion caused the $R Q$ average of $1.01 \pm 0.24$, and the average exercise duration was of $20.6 \pm 9.7 \mathrm{~min}$. There were no significant differences in the $R Q$ values or exercise duration among the three interventions $(p=0.697$ and $p=0.598$, respectively). Caffeine did not increase lipid oxidation or performance of young male individuals.
\end{abstract}

Keywords: coffee, aerobic exercise, ergogenic aids. 


\section{INTRODUÇÃO}

A cafeína tem sido utilizada com grande frequência como substância ergogênica de forma aguda, previamente à realização de exercícios físicos, com o intuito de protelar a fadiga e, consequentemente, aprimorar o desempenho físico, sobretudo em atividades de média e longa duração(1-3). A utilização da cafeína tem se tornado mais comum nos últimos anos, particularmente por atletas de ciclismo e corredores de média e longa distância, devido à suposta ação ergogênica desta substância em esportes de longa duração( ${ }^{(4)}$. Estudos têm apontado para uma melhoria no desempenho atlético em diferentes tipos de exercício físico após a ingestão de 3 a $6 \mathrm{mg}$ de cafeína por quilograma de peso corporal, tanto em atletas amadores, como em atletas de elite ${ }^{(5,6)}$; entretanto, outros falham em encontrar resultado semelhante $\mathrm{e}^{(7,8)}$.

Segundo Yeo et al. ${ }^{(9)}$, associar o carboidrato à cafeína poderia potencializar o efeito ergogênico durante o exercício. Este efeito seria resultado de um aumento na disponibilidade da glicose, efeito oposto ao observado durante o repouso com a co-ingestão de cafeína e carboidrato. Pedersen et al. ${ }^{(10)}$ verificaram aumento na taxa de ressíntese do glicogênio muscular pós-exercício quando os voluntários ingeriam cafeína com carboidrato no período de recuperação de um exercício exaustivo, quando comparado à ingestão de carboidrato. Entretanto, estes achados foram observados em indivíduos treinados e no momento após o exercício, não tendo sido testada esta hipótese nos momentos antes e durante a prática esportiva. Battram et al. ${ }^{(11)}$ sugerem que, em indivíduos destreinados, outros fatores poderiam afetar a síntese de glicogênio muscular pós-exercício (por exemplo, mecanismos independentes de insulina ou baixos níveis de glicogênio na exaustão), podendo acarretar efeitos negativos sobre o metabolismo da glicose.

Consumida como ingrediente em alimentos, bebidas e outras preparações, a cafeína (1,3,7 trimetilxantina) é um componente comum na dieta de muitos atletas. Além de seu uso social, a cafeína tornou-se potencialmente aberta ao emprego incorreto no esporte devido a sua aparente propriedade ergogênica e sua remoção da lista proibida da World Anti-doping Agency (Wada). Certamente, a expansão do mercado das bebidas cafeinadas e de energia no último ano tem sido concomitante para a sustentação de vários esportes por tais companhias de bebidas $^{(6)}$.

Embora tenhamos disponíveis na literatura um número significativo de estudos avaliando os efeitos ergogênicos da cafeína sobre o desempenho aeróbico, ainda não existe consenso entre os autores. Fatores como a intensidade do exercício, a quantidade de cafeína oferecida e o tempo de oferta do produto antes do exercício podem influenciar na sua ação sobre o desempenho. Adicionalmente, a ausência de um grupo placebo nos estudos dificulta a especulação dos resultados em relação aos efeitos da cafeína. Com isso, torna-se importante esclarecermos os efeitos deste tipo de recurso quando comparados a uma mesma situação, entretanto, sem o produto ativo. O objetivo do presente estudo é avaliar o efeito do consumo agudo de cafeína sobre parâmetros metabólicos e de desempenho em indivíduos do sexo masculino, em três situações diferentes.

\section{MÉTODOS}

\section{Amostra}

A amostra foi composta por 15 voluntários do sexo masculino, com idade entre 18 e 30 anos, não engajados em programas de treinamento com finalidades competitivas e não consumidores habituais de bebidas que contenham cafeína (consumo inferior a $100 \mathrm{mg} / \mathrm{dia})^{(12)}$. Todos os indivíduos assinaram termo de consentimen- to livre e esclarecido em duas vias, e o presente estudo foi aprovado pelo Comitê de Ética em Pesquisa da Rede Metodista de Educação do Sul (protocolo n 002/2008). As mulheres não foram testadas devido às alterações hormonais decorrentes do seu ciclo menstrual, pois a fase lútea pode promover alterações no metabolismo da cafeína ${ }^{(3)}$. O cálculo do tamanho amostral foi realizado com auxílio do software Epi-Info considerando-se um poder estatístico de 80\% e um valor a de $5 \%$.

\section{Procedimento para coleta de dados}

Todos os voluntários realizaram anamnese inicial contendo questões sobre suas atividades diárias, hábitos alimentares e consumo de alimentos e bebidas com cafeína. Todos os voluntários preencheram registro alimentar de três dias para verificar a quantidade de cafeína habitualmente ingerida na dieta (Dietwin Profissional ${ }^{\oplus}$ ). Foi solicitado aos voluntários que não modificassem seus hábitos alimentares durante o protocolo do estudo, bem como que não consumissem suplementos alimentares ou bebidas alcoólicas. Caso fosse necessário tomar algum medicamento, as coletas estariam suspensas durante este período até uma semana após o término do período de administração do fármaco. Foram agendadas quatro novas visitas ao laboratório, para a realização do teste máximo de exercício (teste preliminar) e de três testes submáximos com a intervenção dietética (testes experimentais).

\section{Teste preliminar}

Inicialmente, os indivíduos realizaram um teste de cargas progressivas em esteira rolante até a exaustão. Após aquecimento de três minutos caminhando a $5 \mathrm{~km} / \mathrm{h}$ na esteira, aumentou-se a velocidade da esteira em $0,5 \mathrm{~km} / \mathrm{h}$ a cada 30 segundos. $O$ teste foi interrompido após solicitação voluntária do indivíduo, no momento em que ele não possuísse capacidade em manter o exercício. $\mathrm{OVO}_{2 \text { máx }}$ foi determinado com a utilização do equipamento de ergoespirometria da marca VO 2000 (Medgraphics, EUA), e da esteira rolante da marca Imbramed (Millennium ATL, Brasil). Durante todo o teste foram obtidos os valores de consumo de oxigênio $\left(\mathrm{VO}_{2}\right)$, da produção de $\mathrm{CO}_{2}\left(\mathrm{VCO}_{2}\right)$, e da ventilação (VE). A frequência cardíaca (FC) foi obtida através de um monitor de frequência cardíaca da marca Polar (modelo S610, EUA). A partir dos resultados, foram determinados os limiares ventilatórios (LV) e o $\mathrm{VO}_{2 \text { máx }}$ determinado como o maior valor obtido em períodos de 30 s durante o teste.

\section{Testes experimentais}

Cada voluntário realizou três testes submáximos em esteira rolante na intensidade de 10\% abaixo do segundo LV. Trinta minutos antes de cada exercício, os voluntários ingeriram uma das três diferentes soluções determinadas através de um sorteio prévio, que consistiam de: 1) bebida constituída de $5 \mathrm{mg} / \mathrm{kg}$ de massa corporal de cafeína no mesmo sabor da solução placebo, adicionada de água e açúcar (União ${ }^{\oplus}$ ) em concentração de 5\% (café com açúcar); 2) bebida constituída de $5 \mathrm{mg} / \mathrm{kg}$ de massa corporal de cafeína no mesmo sabor da solução placebo, adicionada de água e adoçante (café com adoçante); 3) bebida placebo, que consistia em bebida descafeinada, com sabor idêntico ao café (Iguaçú ${ }^{\oplus}$ ), com adoçante (sacarina sódica e ciclamato de sódio, Zero $\mathrm{Ca}^{\oplus}{ }^{\circledast}$ ) (placebo). Todas as bebidas foram preparadas pelo mesmo pesquisador e os voluntários não tinham conhecimento do conteúdo da bebida que estavam ingerindo.

O protocolo de exercício consistiu de um período de incremento de velocidade da esteira até a estabilização da carga alvo, com cerca de 10 minutos de duração. Após este período de adaptação, a carga foi mantida até a exaustão. Durante todo o período, o indivíduo foi monitorado pelo ergoespirômetro e frequencímetro. 


\section{Análise estatística}

Os dados foram estruturados e analisados utilizando o pacote estatístico SPSS (Statistical Package for Social Sciences) versão 13.0 para Windows, e os resultados foram expressos em média \pm desvio padrão. Avaliou-se a distribuição de todas as variáveis contínuas para a verificação do pressuposto da normalidade, através do teste de Shapiro-Wilks, e a análise da homocedasticidade das variâncias através do teste de Levene. Uma vez aplicado o protocolo de estudo, as variáveis tempo de exercício, FC e QR, nos diferentes momentos de testes, foram comparadas com Anova fatorial e teste post hoc de Tukey. O nível de significância aceito foi de $p<0,05$.

\section{RESULTADOS}

Os dados apontam uma amostra estudada composta por indivíduos jovens, eutróficos ${ }^{(13)}$ e fisicamente ativos ${ }^{(14)}$. Através do cálculo do registro alimentar de três dias, observou-se que os indivíduos não eram usuários habituais de cafeína. Os resultados descritivos estão expressos na tabela 1.

Tabela 1. Descrição da amostra.

\begin{tabular}{l|c}
\hline & Média $\pm \mathbf{d p}$ \\
\hline Idade (anos) & $22,33 \pm 2,76$ \\
\hline Peso $(\mathrm{kg})$ & $71,31 \pm 10,18$ \\
\hline Estatura (cm) & $174,86 \pm 6,05$ \\
\hline Índice de Massa Corporal $\left(\mathrm{kg} / \mathrm{m}^{2}\right)$ & $23,27 \pm 2,66$ \\
\hline Consumo habitual cafeína $(\mathrm{mg} / \mathrm{dia})$ & $60,74 \pm 35,66$ \\
\hline VO $_{2 \text { máx }}(\mathrm{ml} . \mathrm{kg} \cdot \mathrm{min})$ & $50,18 \pm 9,91$ \\
\hline $\mathrm{FC}_{\text {máx }}(\mathrm{bpm})$ & $188 \pm 7,24$ \\
\hline
\end{tabular}

Na tabela 2 estão descritos os dados dos testes experimentais. Não houve diferença significativa em nenhuma variável metabólica com a ingestão das diferentes bebidas.

Tabela 2. Dados dos testes experimentais obtidos após o consumo das três diferentes bebidas (média $\pm \mathrm{dp}$ ).

\begin{tabular}{l|c|c|c|c}
\hline & $\begin{array}{c}\text { Café com } \\
\text { açúcar }\end{array}$ & $\begin{array}{c}\text { Café com } \\
\text { adoçante }\end{array}$ & Placebo & $\mathbf{p}$ \\
\hline $\mathrm{VO}_{2 \text { médio }}$ (ml.kg.min) & $36,63 \pm 7,90$ & $40,82 \pm 12,78$ & $36,57 \pm 11,95$ & 0,498 \\
\hline $\mathrm{VO}_{2 \text { máx }}$ (ml.kg.min) & $46,60 \pm 9,63$ & $51,53 \pm 13,47$ & $46,62 \pm 12,57$ & 0,636 \\
\hline $\mathrm{RQ}$ médio & $0,96 \pm 0,20$ & $0,98 \pm 0,18$ & $1,01 \pm 0,24$ & 0,459 \\
\hline $\mathrm{RQ}$ máx & $1,30 \pm 0,57$ & $1,20 \pm 0,24$ & $1,22 \pm 0,28$ & 0,320 \\
\hline Fc média (bpm) & $170,18 \pm 6,73$ & $166,06 \pm 14,54$ & $168,11 \pm 9,70$ & 0,787 \\
\hline Fc máx (bpm) & $179,60 \pm 9,20$ & $176,46 \pm 13,65$ & $177,67 \pm 9,94$ & 0,119 \\
\hline $\mathrm{VCO}_{2 \text { médio }}$ (ml.kg.min) & $34,76 \pm 6,17$ & $39,10 \pm 11,74$ & $34,79 \pm 7,45$ & 0,703 \\
\hline $\mathrm{VCO}_{2 \text { máx }}$ (ml.kg.min) & $42,25 \pm 7,39$ & $46,86 \pm 12,98$ & $42,81 \pm 9,08$ & 0,838 \\
\hline Valor de pobtido através do teste de Anova fatorial. & & &
\end{tabular}

Valor de $\mathrm{p}$ obtido através do teste de Anova fatorial.

O gráfico 1 apresenta a média de tempo que os voluntários permaneceram em exercício após a ingestão de cada bebida. Embora os voluntários tenham permanecido, em média, menos tempo em exercício quando ingeriram a bebida descafeinada, esta diferença não foi estatisticamente significativa.

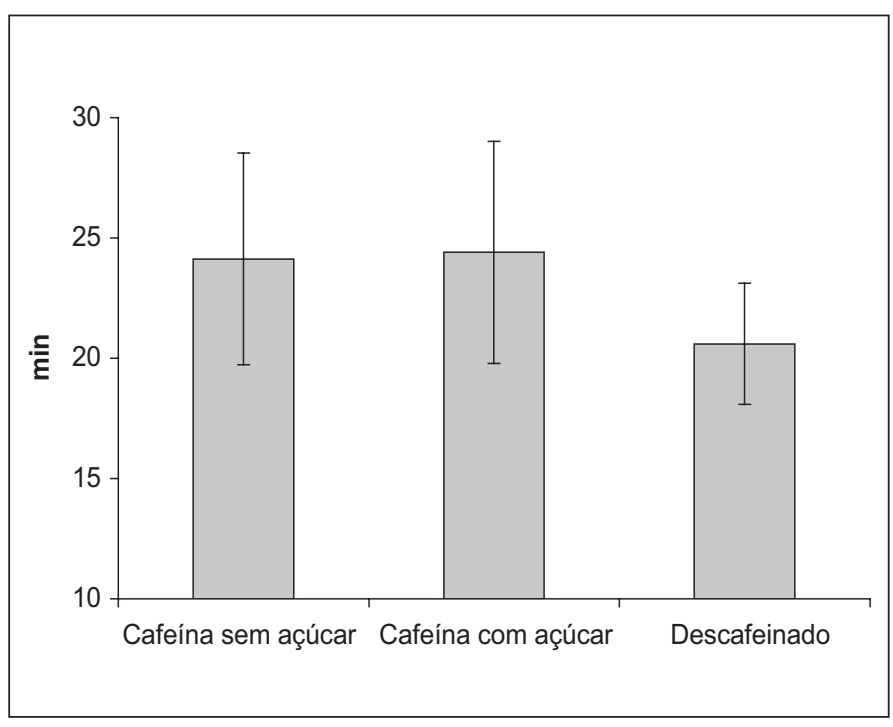

Gráfico 1. Tempo que os indivíduos permaneceram em exercício após o consumo das três diferentes bebidas. Resultados expressos em média $\pm \mathrm{dp}$.

\section{DISCUSSÃO}

O principal achado deste estudo foi que não existe diferença na oxidação de lipídeos do tempo em exercício com a ingestão de cafeína. Por se tratar de um estudo experimental, algumas limitações precisam ser mencionadas. Apesar de ter sido realizada motivação verbal para que todos os voluntários permanecessem em exercício durante maior tempo, outros fatores psicológicos podem influenciar no tempo em exercício de cada voluntário. O uso do bucal para análise dos gases pode ocasionar desconforto ao indivíduo, o que poderia limitar o tempo em exercício. Alguns hormônios também podem alterar a contribuição dos lipídeos como fonte energética e, infelizmente, estes não foram mensurados no estudo. Entretanto, o desenho do estudo utilizando-se de três diferentes bebidas é original e inédito, o que pode contribuir com informações adicionais sobre o assunto.

Os estudos na literatura sobre ingestão de cafeína e desempenho no exercício, em geral são realizados com indivíduos adultos, jovens e saudáveis. Alguns estudos são realizados com atletas ${ }^{(9,10,15-17)}$ e outros com indivíduos fisicamente ativos ${ }^{(7,18-23)}$. Poucos autores utilizam-se da esteira rolante para a realização dos experimentos. Uma possível explicação para isto deve-se ao fato da facilidade que o cicloergômetro oferece para o monitoramento de variáveis metabólicas e bioquímicas. Entretanto, sabemos que a corrida costuma ser a prática mais habitual para as pessoas e, com isso, aumentamos a validade externa do estudo. Ahrens et al. ${ }^{(8)}$ realizaram um estudo submetendo voluntários do sexo feminino ao exercício em esteira rolante na intensidade de $94 \mathrm{~m} \cdot \mathrm{min}^{1}$ ( $3.5 \mathrm{mph}$ ) durante oito minutos, verificando que não houve diferença significativa com relação a frequência cardíaca entre o grupo placebo e o grupo que recebeu $3 \mathrm{mg} / \mathrm{kg}$ de peso de cafeína. Contudo, no grupo que recebeu $6 \mathrm{mg} / \mathrm{kg}$ de peso de cafeína houve aumento nas taxas de dispêndio de energia $(0,23 \mathrm{kcal} / \mathrm{min})$. Malek et al. ${ }^{(7)}$ também realizaram um estudo utilizando esteira rolante para avaliar os efeitos do uso de cafeína durante um período de treinamento. Os autores suplementaram os voluntários que realizavam exercício aeróbico três vezes por semana, oferecendo $201 \mathrm{mg} /$ dia de cafeína, durante oito semanas. O exercício era realizado na intensidade de $75 \%$ do $\mathrm{VO}_{2 \text { máx }}$ e, após o tempo de treinamento, não foram encontrados efeitos ergogênicos com a suplementação de cafeína nos parâmetros analisados. 
Cabe lembrar que os protocolos de suplementação de cafeína antes do exercício diferem bastante entre os estudos disponíveis na literatura. Beaven et al.(15), em um estudo com jogadores profissionais de rúgbi, utilizaram cafeína nas doses de 200, 400 e 800mg/dia e cápsulas de placebo contendo lactose uma hora antes de uma sessão de exercício de resistência. Lorino et al.(1) realizaram um estudo com indivíduos jovens do sexo masculino utilizando $6 \mathrm{mg} / \mathrm{kg}$ de peso de cafeína ou placebo contendo dextrose, ambos em formato de cápsulas gelatinosas. Utilizando cafeína na dose de $5 \mathrm{mg} / \mathrm{kg}$ de peso ou cápsulas de placebo contendo apenas água, Olcina et al. ${ }^{(22)}$ realizaram um estudo com 20 participantes do sexo masculino. Diferentemente, Jacobson et al.(24) realizaram um estudo com ciclistas do sexo masculino utilizando $2,6 \mathrm{~g} / \mathrm{kg}$ de peso de carboidrato ou $2,6 \mathrm{~g} / \mathrm{kg}$ de peso de carboidrato mais $6 \mathrm{mg} / \mathrm{kg}$ de peso de cafeína ou $1,2 \mathrm{~g} / \mathrm{kg}$ de peso de gordura saturada ou $1,2 \mathrm{~g} / \mathrm{kg}$ de peso de gordura saturada mais $6 \mathrm{mg} / \mathrm{kg}$ de peso de cafeína. No presente estudo, utilizou-se três soluções distintas, anteriormente citadas, compostas por café sem açúcar, café com carboidrato (açúcar) e café descafeinado. Deve-se ressaltar que o uso de café descafeinado como protocolo de estudo ainda não havia sido utilizado em pesquisas de outros autores, que realizam com frequência a suplementação na forma de cápsulas, sendo o presente estudo original e inédito por fazer uso desse material ainda não explorado.

A maioria dos estudos disponíveis na literatura oferece cafeína com uma hora de antecedência do exercício aos voluntários, sendo que o tempo de administração de 30 minutos de antecedência pode ter influenciado o resultado do presente estudo. Entretanto, optou-se por utilizar este intervalo de tempo antes do exercício devido ao fato que o pico de concentração da cafeína na corrente sanguínea ocorre em torno de 20 minutos após sua ingestão ${ }^{(25)}$.

No presente estudo, a cafeína não alterou os parâmetros de desempenho nos voluntários. Malek et al.(7) também não verificaram efeito ergogênico com a suplementação de cafeína em um estudo com acompanhamento de oito semanas. Os voluntários treinaram corrida três vezes por semana em intensidade de $75 \%$ do $V_{2} O_{2 \text { áx }}$ e o uso de suplementos à base de cafeína ( $201 \mathrm{mg} / \mathrm{dia}$ ) não aumentou o desempenho dos indivíduos quando comparados àqueles que utilizaram placebo. Entretanto, os dados disponíveis na literatura são contraditórios. Yeo et al. ${ }^{(9)}$ verificaram efeito positivo com a suplementação de cafeína e carboidrato $(5 \mathrm{mg} / \mathrm{kg}$ de peso corporal de cafeína e $5,8 \%$ de glicose). Os indivíduos foram submetidos ao exercício no cicloergômetro na intensidade de $65,7 \%$ do $\mathrm{VO}_{2 \text { máx }}$ durante 120 minutos. Verificando que a glicose, quando ingerida juntamente com a cafeína, resulta em maior oxidação exógena de carboidrato quando comparada à ingestão de carboidrato apenas. Silveira et al.(23) obtiveram efeito ergogênico com a suplementação de $5 \mathrm{mg} / \mathrm{kg}$ de peso de cafeína 60 minutos antes da sessão de exercício em cicloergômetro na intensidade de 30\% acima do limiar anaeróbico, verificando aumento da lipólise induzida pelo consumo de cafeína.

Embora tenha sido observado um valor de QR maior quando os indivíduos ingeriram a bebida descafeinada e um valor mais baixo de QR quando os indivíduos ingeriram a bebida com açúcar, estas observações não foram estatisticamente significativas. Ao contrário de nossos achados, Silveira et al. ${ }^{(23)}$ observaram aumento da lipólise 60 minutos após o consumo de $5 \mathrm{mg} / \mathrm{kg}$ de peso corporal de cafeína, provavelmente devido à maior disponibilidade de lipídios plasmáticos antes do exercício, analisados através de coleta de amostras de sangue imediatamente antes de cada sessão de exercício. Não foi possível verificar se houve aumento da concentração de ácidos graxos livres antes do exercício, pois o presente estudo não realizou coletas sanguíneas para analisar tal parâmetro. Talvez se o tamanho da amostra do presente estudo fosse maior, poderia ter-se observado diferenças significativas no QR entre os grupos. Entretanto, preferiu-se respeitar o cálculo do tamanho amostral previamente realizado e, assim, evitar um viés de seleção na interpretação dos resultados.

Analisando o tempo em que os indivíduos permaneceram em exercício após o consumo das três diferentes soluções, observamos diferença não significativa com relação à maior permanência em exercício na utilização das bebidas compostas somente por cafeína ou cafeína com carboidrato. Utilizando igualmente o parâmetro de tempo em exercício para analisar o efeito da cafeína, Bell e Mclellan ${ }^{(21)}$ utilizaram a dosagem de $5 \mathrm{mg} / \mathrm{kg}$ de peso de cafeína 1,2 e três horas antes de exercício em cicloergômetro na intensidade de $80 \%$ do $\mathrm{VO}_{2 \text { máx }}$ em dois grupos, classificados como usuários e não usuários de cafeína. Ao término do estudo, verificou-se que os indivíduos não usuários de cafeína permaneceram mais tempo em exercício, possivelmente devido à sensibilidade à cafeína.

A adição de carboidrato à bebida cafeinada tem sido utilizada por alguns autores como metodologia de estudo(9,10,24,26). A diminuição de utilização dos níveis de glicogênio muscular e o aumento da produção de glicogênio hepático parecem ser os mecanismos envolvidos com a utilização de carboidrato como recurso ergogênico. Yeo et al.(9) utilizaram três diferentes soluções durante exercício em cicloergômetro (glicose na concentração de 5,8\%, glicose na mesma concentração com $5 \mathrm{mg} / \mathrm{kg}$ de peso corporal de cafeína, ou placebo, composto somente por água). Os autores verificaram que a ingestão de cafeína e carboidrato durante ou após o exercício resultou em maior ressíntese de glicogênio muscular e maior oxidação de carboidrato após o exercício. Nossos resultados não apontaram diferenças significativas nos parâmetros analisados quando adicionamos carboidrato na bebida oferecida. A explicação para tal fato é que a adição de carboidrato à bebida cafeinada foi útil para manter a glicemia durante o teste, através do aumento dos níveis de glicogênio hepático, pois o uso de carboidrato não gera um aumento do tempo em exercício.

Em conclusão, este estudo demonstrou que a ingestão de 5mg/ $\mathrm{kg}$ de peso de cafeína, adicionada ou não de carboidrato e oferecida 30 minutos antes do exercício aeróbico, não aumentou a oxidação de lipídios nem o tempo em exercício. Entretanto, o desenho do estudo é original, oferecendo a suplementação de cafeína através de uma bebida que pode facilmente ser preparada na residência do indivíduo, além da utilização de uma bebida descafeinada ao invés de cápsulas de placebo. Mais estudos sobre a utilização de cafeína como potente recurso ergogênico no exercício aeróbico são necessários, para que estes aspectos controversos acerca dos mecanismos responsáveis pela sua eficácia e do protocolo de suplementação para ocasionar os efeitos ergogênicos sejam esclarecidos.

Todos os autores declararam não haver qualquer potencial conflito de interesses referente a este artigo. 


\section{REFERÊNCIAS}

1. Lorino AJ, Lloyd LK, Crixell SH, Walker JL. The effects of caffeine on athletic agility. J Strength Cond Res 2006;20:851-4.

2. McClaran SR, Wetter TJ. Low doses of caffeine reduce heart rate during submaximal cycle ergometry. J Int Soc Sport Nutr 2007;4:1-9.

3. Molt RW, O'Connor PJ, Dishman RK. Effect of Caffeine on Perception of Leg Muscle Pain During Moderate Intensity Cycling Exercise. The Journal of Pain 2003;4:316-21.

4. Thuyne VW, Delbeke FT. Distribution of Caffeine Levels in Sport Urine in Different Sports in Relation to Doping Control Before and After the Removal of Caffeine from the WADA Doping List. Int J Sports Med 2006;27:745-50,

5. Altimari LR, Melo JC, Trindade MCC, Cyrino ES, Tirapengui J. Cafeína e exercício físico aeróbico. Soc Bras Alim Nutr 2006;31:76-96.

6. Chester N, Wojek, N. Caffeine Consumption Amongst British Athletes Following Changes to the 2004 WADA Prohibited List. Int J Sports Med 2007;29:524-8.

7. Malek MH, Housh TJ, Coburn JW, BeckTW, Schmidt RJ, Housh DJ, et al. Effects of eight weeks of caffeine supplementation and endurance training on aerobic fitness and body composition. J Strength Cond Res 2006;20:751-5.

8. Ahrens JN, Crixell SH, Lloyd LK, Walker JL. The physiological effects of caffeine in womem during treadmill walking. J Strength Cond Res 2007;21:164-8.

9. Yeo SE, Jentjens, RLPG, Wallis GA, Jeukendrup AE. Caffeine increases exogenous carbohydrate oxidation during exercise. J Appl Physiol 2005;99:844-50.

10. Pedersen DJ, Lessard SJ, Coffey VG, Churchley EG, Wotton AM, Ng T, Watt MJ et al. High rates of muscle glycogen resynthesis after exhaustive exercise when carbohydrate is coingested with caffeine J Appl Physiol 2008;105:7-13

11. Battram DS, Shearer J, Robinson D, Graham TE. Caffeine ingestion does not impede the resynthesis of proglycogen and macroglycogen after prolonged exercise and carbohydrate supplementation in humans. J Appl Physiol 2004;96:943-50.

12. Griffiths RR, Evans SM, Heishman SJ, Preston KL, Sannerud CA, Wolf B, et al. Low-doses caffeine physical dependence in humans. J Pharmacol Exp 1990;255:1123-32.
13. World Health Organization. Preventing and managing the global epidemic. Report of a WHO Consultation on Obesity. WHO, Geneva, 1998.

14. American College of Sports Medicine. Manual do ACSM para avaliação da aptidão física relacionada à saúde. Rio de Janeiro: Guanabara-Koogan, 2006.

15. Beaven CM, Hopkins WG, Hansen KT, Wood MR, Cronin JB, Lowe TE. Dose Effect of Caffeine on Testosterone and Cortisol Responses to Resistance Exercise. Int J Sport Nutr Exerc Metab 2008;18:131-41.

16. Conway KL, Orr R, Stannard SR. Effect of a divided caffeine dose on endurance cycling performance, postexercise urinary caffeine concentration, and plasma paraxanthine. J Appl Physiol 2003;94:1557-62.

17. Jenkins NT, Trilk JL, Singhal A, O'Connor PJ, Cureton KJ. Ergogenic Effects of Low Doses of Caffeine on Cycling Performance. Int J Sport Nutr Exerc Metab 2008;18:328-42.

18. Gliottoni RC, Motl RW. Effect of Caffeine on Leg-Muscle Pain During Intense Cycling Exercise: Possible Role of Anxiety Sensitivity. Int J Sport Nutr Exercise Metab 2008;18:103-15.

19. Norager $C B$, Jensen $M B$, Madsen MR, Laurberg S. Caffeine improves endurance in 75-yr-old citizens: a randomized,double-blind, placebo-controlled, crossover study. J Appl Physiol 2005;99:2302-6

20. Tarnopolski M, Cupido C. Caffeine potentiates low frequency skeletal muscle force in habitual and nonhabitual caffeine consumers. J Appl Physiol 2000;89:1719-24

21. Bell DG, Mclellan TM. Exercise endurance 1, 3, and $6 \mathrm{~h}$ after caffeine ingestion in caffeine users and nonusers. J Appl Physiol 2002;93:1227-34.

22. Olcina GJ, Timón R, Muñoz D, Maynar Jl, Caballero MJ, Maynar M. Caffeine ingestion effects on oxidative stress in a steady-state test at 75\%VO2max. Science \& Sports 2008;23:87-90.

23. Silveira $L R$, Alves $A A$, Denadai BS. Efeito da lipólise induzida pela cafeína na performance e no metabolismo de glicose durante o exercício intermitente. R Bras Ci e Mov 2004;12:21-6.

24. Jacobson TL, Febbraio MA, Arkinstall MJ, Hawley JA. Effect of caffeine coingested with carbohydrate or fat on metabolism and performance in endurance trained men. Exp Physiol 2006;86:137-44.

25. Sinclair CJ, Geiger JD. Caffeine use in sports: a pharcological review. J Sports Med Phys Fitness 2000;40:71-9.

26. Kovacs EMR, Stegen JHC, Brouns f. Effect of caffeinated drinks on substrate metabolism, caffeine excretion, and performance. J Appl Physiol 1998;85:709-15. 